\title{
Optimization of product line design for environmentally conscious technologies in notebook industry
}

\author{
*K. H. Lin; L. H. Shih; S. C. Lee \\ Department of Resources Engineering, National Cheng Kung University, No.1, University Road, \\ Tainan, 701, Taiwan \\ Received 11 November 2009; $\quad$ revised 1 February 2010; accepted 4 May 2010; availaE@Honline 1 June 2010
}

\begin{abstract}
Promotion of green technologies related to notebook computer will have significant benefits in the environment. Notebook companies need to make a careful market assessment for green technologies. Due to the variety of consumer preferences for green technologies, as well as a hot competitive climate in notebook market, consumer preferences should be taken into consideration during the assessment process. This study classifies the green technologies of notebook industry. Some green technologies are not controlled by the environmental regulations but are popular among customers. This study named this kind of technologies niche green technologies. The product line design model can evaluate the design scheme based on customer preferences. Therefore, this study uses conjoin analysis to investigate the consumers' preferences for assorted technology. Subsequently, product line design model is utilized to seek the optimal scheme of niche green technologies adoption based on the consumers' preference. Results of conjoint analysis reveal that consumers value two attributes, including price and size. Furthermore, the preferences for niche green technologies in solid state drive disk and light emitting diode backlight surpass the former technology. After the assessment of market situation with product line design model, two types of niche green technologies, including lithium polymer battery and light emitting diode backlight are suggested for the adoption of new products design.
\end{abstract}

Keywords: Conjoint analysis; Consumer preferences; Environmental regulations; Green technologies; Notebookcomputer

\section{INTRODUCTION}

Because of increasing worldwide awareness of sustainable development, more and more consumers are leaning towards purchasing green concept products. In the notebook computer (NB) industry, development and adoption of new technologies has been fairly frequent, including the new green technologies (GT). For the NB corporations in Taiwan that are trending away from the original equipment manufacturer (OEM) business and towards ordnance bench mark (OBM), introducing green products will improve their brand-name recognition. Besides, the complete product design and product line extension can benefit market share and improve profitability (Chien and Shih, 2007; Huang and Shih, 2009). In worldwide NB industrials, Taiwan has played an important role in research and development, design, manufacturing and marketing and should therefore become the forerunner in Niche GT. There is a host of factors prompting companies to design and

*Corresponding Author Email: n4892126@ccmail.ncku.edu.tw Tel.: +886 06275 7575; Fax: +88606 2380421 develop green technologies and products with the main factor relying on the sustainable development and environmental awareness of the consumer (Hsu and Hu, 2008; Tuzkaya and Gulsum, 2008; Chen, 2009; Tehrani et al., 2009). With the proliferation of NB products, the development of relevant GT is very important, especially Niche GT which international protocols do not enforce but help to save resource and energy. Most consumers do not have special preference to Niche GT, but maybe some consumers will prefer to purchase the product which uses above technologies. The strategy of applying Niche GT to the NB launched in Taiwan is worth studying. Under the consideration of cost and consumer preferences, how to match Niche GT with a product line is an interesting and challenging issue.

Niche GT give more choices for consumers. Consequently, companies need to review their existing product line. Many researchers have studied whether existing product line needs to be changed on the basis of consumers' preference. Conjoint analysis (CA) is 
used to measure the consumers' preferences of NB products. Niche GT become new levels of product attributes in this study. The product line design model (PLDM) is then used to find the optimal product line extension scheme (PLES) that adopt Niche GT. This study measures the consumer preferences to simulate purchase tendencies of consumers and finds out the optimal PLES for Example Company Optimal product line design is one of the key problems in marketing (Green and Krieger, 1991; Szymanski et al., 1993; Alexouda, 2002, 2005). Many researchers have proposed some methodologies based on consumer preferences (Alexouda and Paparrizos, 1999, 2001; Li and Azarm, 2002; Steiner and Hruschka, 2003; Balakrishnan et al., 2004; Bandyopadhyay and Chattopadhyay, 2007; Nnorom and Osibanjo, 2009). In this problem, products that have entered the market before the introduction of a new product line are termed existing products. Individual customers switch from existing products only if new products provide more utility. Zufryden (1979) and Kohli and Sukumar (1990) formulated the product line design problem as a 0-1 integer program and demonstrated that the problem is NP-Hard. Alexouda and Paparrizos (2001) and Steiner and Hruschka (2003) applied genetic algorithms (GA) to solve this problem. Conjoint analysis is a very popular method that has had thousands of commercial applications. Green and Krieger (1985); Dobson and Kalish (1988); Kohli and Sukumar (1990) and Nair et al., (1995) have used conjoint methods for product line design problem. Based on CA, a small number of different product profiles are tested and the part-worth utility obtained from each level of different attributes is estimated. In this way, a matrix of consumers' partworth utilities is estimated. Based on these part-worth utilities, the utilities of the candidate products can be computed (Green and Krieger, 1989).

Taiwan has played an important role in worldwide NB product $\mathrm{R}$ and $\mathrm{D}$, design, manufacturing and marketing and should therefore become the forerunner in the Niche GT issue. This is the reason behind this study on the NB industry in Taiwan. In summary, this study takes the consumer part-worth utilities obtained from CA as the foundation and then the utility data are used by PLDM to evaluate the optimal PLES of NB. This study focuses on the selection of Niche GT with most potential. These technologies became the new levels for each attribute of NB product. Then CA can be applied to evaluate consumer preferences. The partworth utilities can simulate the purchase tendency of consumer and can highlight the preferences and weight of Niche GT in the consumer's mind.As the PLDM is an NP-hard problem, genetic algorithm is used to obtain the appropriate PLES.

This research has been done in National Cheng Kung University, in Tainan, Taiwan, from July, 2008 to October, 2009.

\section{MATERIALS AND METHODS}

Classification of GT

The main concerns for adoption of GT in the products include environmental regulation and technology maturity. Therefore, this research categorizes GT based on these two factors, as shown in Fig. 1, regulation refers to the environmental regulations or international environmental agreements for GT. In addition, technology maturity signifies the adoption of GT in NB under reasonable cost, without compensating the product quality and security. Subsequently, GT classification matrix categorizes GT into regulation driven, foresight and niche. GT under regulation is divided into immediate adoption and future adoption according to the level of maturity. In the same way, GT not under regulation is divided into foresight and niche by the level of maturity.

\section{Regulation driven}

The upper half of the classification matrix is restrained by environmental regulations. The environmental regulations usually care about immoderate use of hazardous substance and excessive resource dissipation. Then, it is divided into immediate and future in accordance to the replacement by mature GT. For instance, mature GT will be immediately employed due to the regulations. The adoption of Regulation Driven GT is required at an appropriate timing based on the development of the technologies. In addition, most of regulation driven GT are not the main concern of consumers in purchasing NB. Without considerable profit, companies will not actively develop these technologies. Therefore, regulations are needed to compel and drive the development of various GT. With the power of country and market, NB companies are compelled to research, develop and apply mature GT. Besides, appropriate buffer period for immature GT is planned for gradual implementation of research and adoption.

\section{Foresight GT}

Although there are GT unrestrained by regulations, some of the NB companies may voluntarily introduce 
Int. J. Environ. Sci. Tech., 7 (3), 473-484, Summer 2010

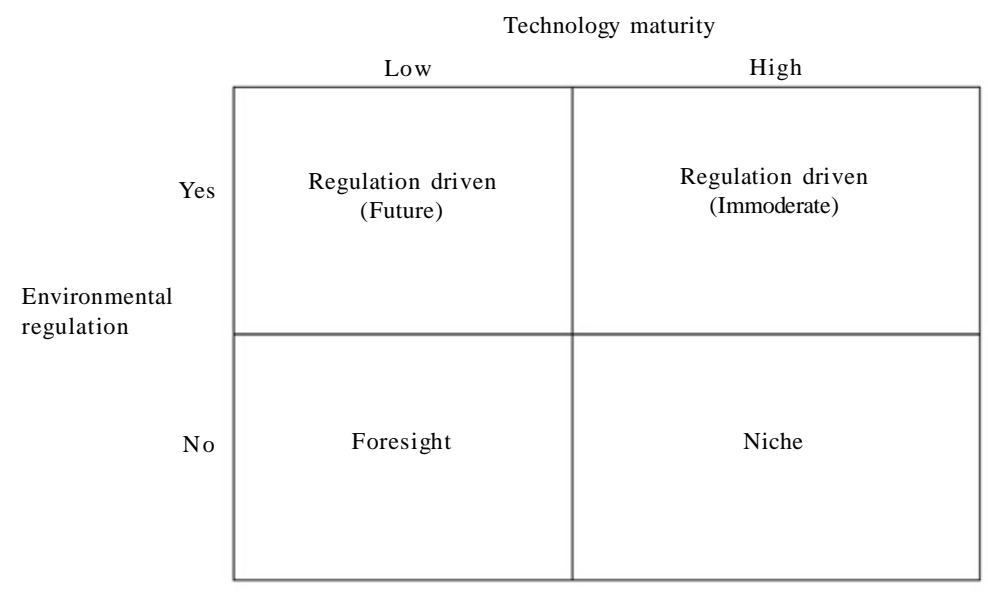

Fig. 1: Classification matrix of GT

GT which are beneficial to the environment. The NB companies may develop these GT for the consideration in environmental protection and market expansion. In the classification matrix, these GT are situated in the lower portion. Furthermore, the lower left of the matrix is foresight GT with market potential but low maturity, which is known as foresight GT. Although this type of immature GT is not applied to the products within a short term period, there are respectable environmental benefit and market potential in this type of GT. Therefore, some companies will invest in research and development of foresight GT.

\section{Niche GT}

Niche GT and foresight GT have similar characteristics as they provide environmental protection and sales of product at the same time. However, Niche GT has higher maturity as compared to foresight GT. Since Niche GT is readily applied in NB products, its contribution to environmental protection and improvement in performance of the products will beckon the consumers from the niche market. Moreover, companies can draft appropriate strategies for Niche GT adoption by analyzing the scale and purchasing power of this consumer group.

Environmental regulations are the motivation for development and adoption of regulation driven GT. In the market, all companies must fulfill specific criteria before they can market their products. Therefore, companies do not easily possess competition advantages in this type of technology. On the other hand, foresight GT is not applied to the products in the presence market due to the immaturity of technology.
Besides, enterprise groups regularly will utilize their abundant research resources to acquire future competition advantages in foresight GT. Additionally; specific consumer group is attracted by Niche GT which has been applied in various products. Companies may gain immediate competition advantages if they can promptly reflect consumers' preferences in the design and manufacturing of their products. For NB companies in Taiwan which are known for the flexibility in product design and manufacturing, proficient utilization of Niche GT in designing PLES can not only expand market but also enhance brand image. Therefore, this study uses PLDM to explore the optimal PLES for NB companies in Taiwan.

\section{Consumer preference}

This research formulates the problem of product line design within a CA framework. Products are described using attributes and attribute levels. CA is a very popular method that has thousands of commercial applications. Several researchers have used the conjoint methods for product line design issues.

Conjoint analysis has been widely used in various problem domains, including product positioning, market segmentation, product line development, pricing and corporate image building (Krieger et al., 2004). Wittink and Cattin (1989) notes that CA has been used to solve product marketing problems for more than 400 products. Green and Srinivasan (1978) summarize six parts of a CA as: 1) preference model, 2) data collection method, 3) stimulus set construction, 4) stimulus presentation, 5) measurement scale for the dependent variable and 6) estimation method. 
The NB is formulated within the CA framework. Products are described using attributes and attribute levels. A representative buyer population sample is used to estimate the part-worth utility of each individual for each attribute level. The consumer decision making process is represented through a multi-attribute utility approach in which an individual's utility derived from a product can be computed by adding the part-worth utilities of the selected attribute levels. The consumers purchase the products that provide the most utility.

In CA, the characteristics of a product are described in terms of attributes. Variations within an attribute are described as levels. Attribute levels are the values of the independent variables. An analyst specifies the levels for each attribute and then asks a respondent only for that person's overall preference. In this way, CA can compute the part-worth utility of each attribute level. Large part-worth values are assigned to the most preferred levels and small part-worth values are assigned to the least preferred levels. A linear regression model with conjoint preference data would be:

$Y_{A}=W_{0 A}+\sum_{k=1}^{K} \sum_{j=1}^{J_{k}} W_{j k} X_{A j k}$

Where, $Y_{A}=$ the rating assigned to product profile $A$.

$A=$ product profile.

$W_{0 A}=$ constant term for product profile $A$.

$W_{j k}=$ the utility coefficient for level $j$ on attribute $k$.

$X_{A j k}=$ a dummy variable defined as: $X_{A j k}=1$ if product profilehas levelon attribute; otherwise.

\section{The GA for the PLDM}

The PLDM can be formulated within CA framework as a $0-1$ integer programming problem. Products are described using attributes and attribute levels. Partworth utilities of each attribute for each buyer can be estimated by CA. The PLDM provides a methodology that links attributes directly to consumers' preferences. This model can evaluate what kind of product each consumer intends to choose.

The PLDM will create a scheme for adoption of GT based on the existing product line. This study uses product line design optimization problem of Alexouda (2004) and apply the part-worth utilities in it. The resulting part-worth utilities can predict consumer purchase tendencies. The objective function of PLDM is to maximize the number of consumers who change the purchase tendency from the competitors' product to the candidate products of the PLSE. GA is used as an optimization engine because PLDM is a NP-hard problem. This section applies GA to solve this NPhard problem. In the formal description of the GA, the expected market share that is maintained in matrix TOTAL_SOC $_{\mathrm{p}}$ which is used for the fitness evaluation of the population elements (extension schemes $p \in$ $\Pi)$. Fig. 2 shows the flowchart for fitness evaluation of extension schemes $p \in \Pi$.

Matrix STATUTIK maintains the part-worth utilities of the competitors' existing product which has maximal preference for each consumer $i \in \Theta$; Matrix PRODUTIL $_{I}$ maintains the part-worth utilities of Example Company's existing product which has maximal preference for each consumer ; Let denote the set of the candidate PLES. The population is maintained in a matrix $P O P_{P^{*} I^{*} M}$. The elements $P O P_{p m k}$, where $p \in \Pi$, $m \in \Psi$ and $k \in \Omega$, denote the selected level of each attribute. That is, if level $j \in \Phi_{k}$ of attribute $k \in \Omega$, is assigned to product $m \in \Psi$ of PLES $p \in \Pi$, then $P O P_{p m k}=j$. The initial population of candidate PLES is randomly generated and stored in matrix $P O P_{p^{*} m^{*} k}$.

The fitness of population is evaluated according to the market share maximization criterion. Matrices $A D D P U_{P^{*} I^{*} M}, S O C_{P I}$ and TOTAL_SOC $\mathrm{P}_{\mathrm{P}}$ are computed in order to evaluate the expected market share of the PLES. Matrix ADDPU $U_{P *^{*} M}$, maintains the utilities of the $M$ different products of each candidate PLES which are derived by each consumer. It can be computed as follows: $A D D P U_{p i m}=\sum_{k \in \Omega} W_{i\left(P_{P O P}{ }_{p m k}\right)}$ where $p \in \Pi$, $i \in \Theta, m \in \Psi$.

$S O C_{p i}$ indicates whether consumer $i \in \Theta$ who originally choose competitors' existing product would change to buy one item of the PLES $p \in \Pi$. Let $m^{\prime}$ denote the index $m$ such as $A D D P U_{p i m^{\prime}}=\max _{m \in \Psi}$ $A D D P U_{\text {pim }}$, where $p \in \Pi$ and $j \in \Phi$. In particular, if consumer $i \in \Theta$ whose maximal part-worth utility among the competitors' existing products is higher than the maximal part-worth utility from Example Company's existing products (PRODUTIL $L_{i}<$ $S_{\text {STATTIK }}$ ) and the maximal lower bound part-worth utility from PLES $p \in \Pi$ is higher than the maximal upper bound part-worth utility from competitors' existing products $\left(A D D P U_{\text {pim }},>S T A T U T I K_{i}\right)$, then SOC 


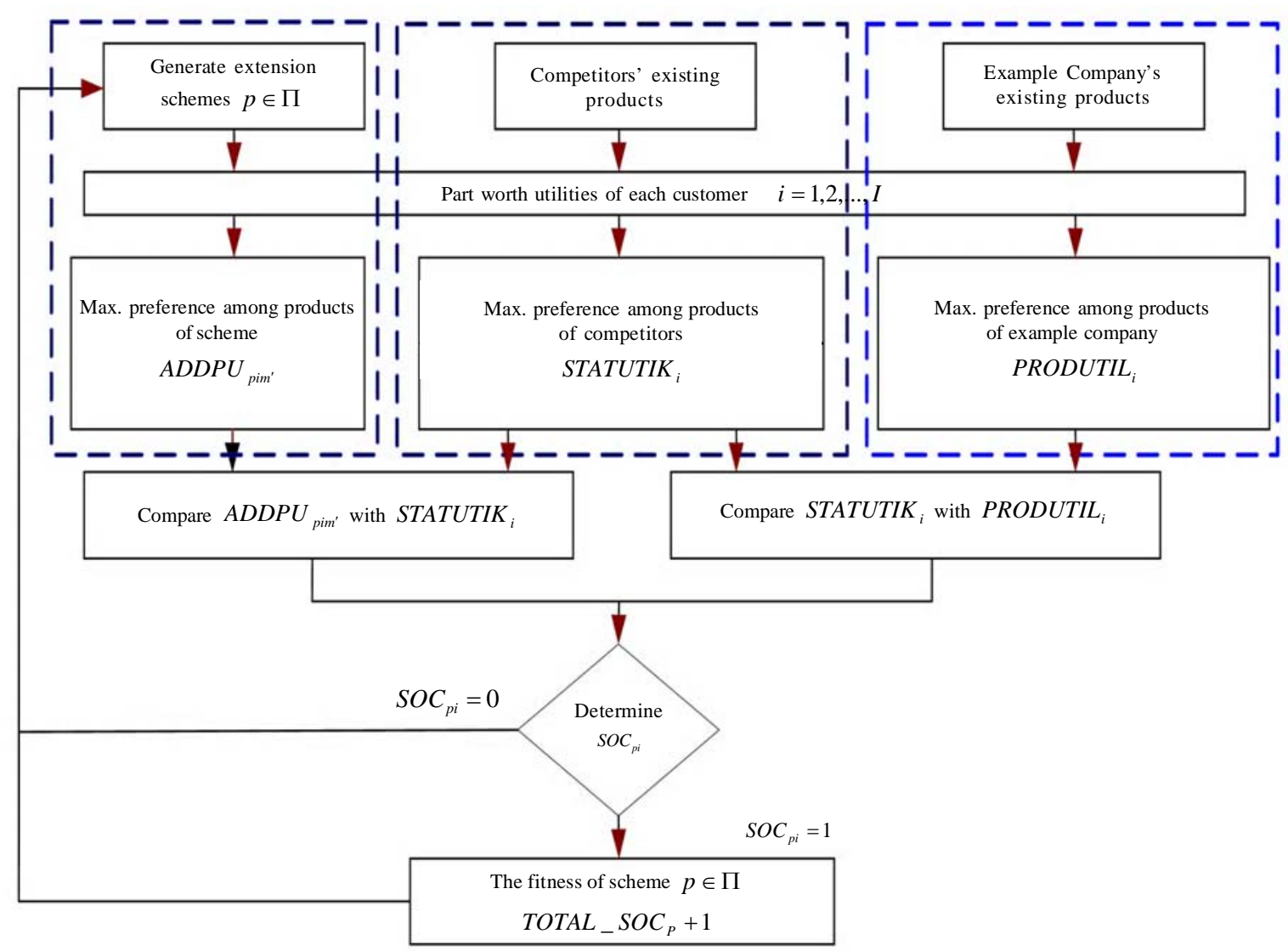

Fig. 2: Flowchart for fitness evaluation of extension schemes

${ }_{p i}=1$, else $S O C_{p i}=0$.

If PRODUTIL $i_{i}<$ STATUTIK $\quad i$

and ADDPU pim $>$ STATUTIK i

then $\quad S_{p i}=1$

else $\quad$ SOC $_{p i}=0, \quad p \in \Pi, \quad i \in \Theta$

The number of consumers of each candidate PLES is stored in matrix TOTAL_SOC , that can be computed as follows:

TOTAL_SOC ${ }_{P}=\sum_{i \in \Theta} S O C_{p i}, \quad$ where $p \in \Pi$

The Niche GT of NB

With the proliferation of NB computers, the development of relevant GT is very important, especially Niche GT which international protocols do not enforce but help to save energy and resources and influence consumers' willingness to buy. This kind of GT that is not required by regulations depends on the support of consumers and companies to become mature and popular. NB industry attach great importance to following GT that can be adopted under reasonable cost, without compensating the product quality and security. The strategy of applying following GT to the NB launched in Taiwan by Acer and Asus is worth studying. Under the consideration of cost and consumer preferences, how to match Niche GT with a product line is an interesting and challenging issue.

\section{Bio-based plastic shell}

Bio-based plastic material was first used to manufacture the packages for some small components in NB. One raw material of polyactic acid, is distilled from corn and other plants. Half of the entire case of the RMV-BIBLO NB80K green NB launched by Fujitsu in Japan in the spring of 2005 is made of bio-based 
plastic. Gross and Kalra (2002) posit that one advantage of this bio-based plastic is to reduce the use of petroleum-based plastic and reduce dependency on oil. Carbon emissions in the life cycle of this kind of product are reduced by $15 \%$. One disadvantage is the high cost of materials, technology limitations and patent licenses; therefore, only Fujitsu is applying this technology to cases and Sony is using the technology for small plastic components at present.

\section{Lithium polymer battery}

A battery's utility is based on weight, shape, endurance, safety and environmental protection. Nowadays, most NB batteries are lithium ion, but short circuits in these batteries can cause thermal runaway effects, hyperthermia and even fires. In recent years, massive recalls of lithium ion batteries manufactured by Sony show the instability of lithium ion batteries, impacting user safety and wasting natural resources. The capabilities and traits of lithium polymer batteries are similar to those of lithium ion batteries. Some references suggest that compared to lithium ion batteries, lithium polymer batteries benefit from good thermal capabilities and production technology. In addition, lithium polymer batteries can be made in as many shapes as consumers like. Masuda et al., (2007) indicates that lithium polymer batteries provide better safety and energy density and can be made light weight and provide long life. Lightness, thinness, material savings and stable life are the most environmental characteristics of lithium polymer batteries.

\section{Light emitting diode (LED) backlights}

Because the technology for cold cathode fluorescent lamps (CCFL) is mature and such products are low cost and provide linear luminescence through symmetrical lamp-housing, this technology is still used as the main backlight for TFT-LCD. The environmental problem of CCFL is that it contains hydrargyrum. Although hydrargyrum is provided an exclusive clause by the EU, it is still a problem for environmental protection. In addition, high power consumption is a key disadvantage of CCFL technology; it is bad for a NB, which needs to scrimp on every iota of power consumption. Tsai (2007) indicates that LED technology provides high resistance to shock, long life and low energy consumption. Given the technology's advantages of energy conservation, elimination of hydrargyrum and lightness and thinness when applied to backlighting, the work group aimed to extend the per-charge life of a NB by urging NB companies to adopt LED backlights. Environmental protection, elimination of hazardous substances and power economy are the key advantages of LED backlights.

\section{Solid state disk (SSD)}

The main disadvantages of standard hard disks are poor resistance to shock, high power consumption and mechanical latency. These three disadvantages are serious problems for NB, which require security of data, power economy and high efficiency. Kang et al., (2003) suggest that solid state disks take less space, are light weight, minimize energy consumption and quickly store and fetch data. In addition, they are highly reliable. All of these characteristics enable the design of NB that are lighter, thinner and power efficient with longer life between battery charges. Solid state disks also cut in half the time needed to shut down and reduce the risk of data loss. The power economy and long life of solid state disks are environmentally friendly characteristics.

\section{Conjoint analytic design for $N B$}

The CA adopted in this study includes six attributes used to grade NB tested for consumers; they are presented in Table 1 . The first row shows the important attributes of NB, and the second row shows the levels of these attributes. Below Table 1, instructions are given for each level of every attribute in detail, and the leading four attributes are used as competitive factors for two kinds of levels of normal technology and GT. The attributes of size and price are key factors influencing for NB purchases and there are 4 and 3 levels respectively. In addition to the six attributes, the other characteristics of NB are considered unchanged. The levels of Case attribute include four levels: plastic, carbon fiber, metal and bio-based plastic. The levels of Batteries attribute include lithium ion and lithium polymer technologies. The levels of backlights attribute include CCFL and LED. The levels of hard disk attribute compare hard disk drives (HDD) and solid state disks. Size and price are two important attributes for NB. By combining these two attributes with the four attributes above, the profiles of NB can be described completely and let the respondents provide their evaluations. 


\section{Solution procedure of proposed GA}

Because the product line design problem is NP-hard, it is impossible to solve real sized problems in realistic time by using methods which guarantee a global optimal solution. Many researchers have proposed heuristic procedures to solve this. Balakrishnan and Jacob (1996) developed a GA for a single product design problem. In recent years, many scholars have used GA for product line design problems. GA is not guaranteed to find a global optimal solution, but it will find a nearly optimal solution in realistic time. Fig. 3 shows the GA solution procedure. In the formal description of the GA, the $40 \%, 40 \%$ and $20 \%$ of the new population elements are produced using the selection method, the crossover operator and the mutation operator, respectively. Selecting these parameters can crucially affect the efficacy of the GA. The proposed GA will stop when the best candidate PLES does not improve in the last 50 consecutive. During the process of analysis with a GA, the chromosomes with high fitness had a higher probability to be chosen to produce the next generation, and this study used a Roulette wheel to choose the program. And $95 \%$ of the chromosomes chosen will produce the next generation by copulating, and the rest of them will retained directly; $5 \%$ of the new generation's chromosomes will mutate and produce chromosomes with new traits. Also, the number of each generation is set as $20(M=20)$ and the washed-up condition is that the optimal solution of the 20 continued generations will not change at all.

\section{RESULTS AND DISCUSSION}

In the past the studies of CA focused on marketing and the studies of PLDM focused on algorithm improvement. In recent years, CA was popular in product design field. This study makes good use of the advantages of CA and integrates with PLDM to find out the optimal product line design scheme for environmentally conscious technologies.

Table 1: Attributes and attribute levels

\begin{tabular}{ll}
\hline Attribute & Level \\
\hline Case & plastic, carbon fiber, metal, bio-based plastic \\
Batteries & lithium ion, lithium polymer \\
Backlights & CCFL, LED \\
Hard disk & HDD, SSD \\
Size & $7,11,14,17$ \\
Price & $20000 \quad 70000 \quad 120000$ \\
\hline
\end{tabular}

The questionnaire was put on a Web site (http:// 140.116.74.4/ox/NB.htm) and announced on several popular NB websites and BBSes to collect data. These websites and BBSes were picked because of their popularity and the large number of visits. After two months, 201 responses were collected via the Web questionnaire and 175 responses were validated. The value of Pearson's $\mathrm{R}$ is 0.956 that represent this model has a good model-fit evaluation. The result of this CA survey can explain the customer preference well. Most participators in this survey are college students and White-collar workers. The ages of them are between twenty and forty. The sample of this study can represent the segment who buy NB by himself, but that does not represent the demands of business and government.

The software for this study was programmed in Visual C++ 6.0 and was used a Pentium (R) 4 (1024MB, $2.80 \mathrm{GHz}$ running Windows $\mathrm{XP}$ ) to run the program. Based on different scales of the product line to calculate, this study ranged five product attributes $(K=5)$ such as case material, battery, backlight, state disk and size. The attribute of price was derived by adding each attribute value after evaluation but not matched randomly. Each attribute has 4, 2, 2, 2 and 10 levels $\left(\mathrm{J}_{1}=4, \mathrm{~J}_{2}=2, \mathrm{~J}_{3}=2, \mathrm{~J}_{4}=2, \mathrm{~J}_{5}=10\right)$ and the number of consumers is $175(\mathrm{I}=175)$.

\section{The relative importance of each attribute}

In comparing the importance of each attribute, Fig. 4 shows that size and price have relatively high importance. The case attribute has the highest importance of the four attributes which include new technologies. This result implies that consumers have a notable preference between the utilities of the levels of the case attribute.

\section{Utility of each attribute level}

Table 2 shows the average part-worth utilities of the 175 responses in this study. The results are summarized as follows:

- Of the four new technologies, the average utilities of LED backlight and SSD hard disk are higher than traditional technologies in backlight and hard disk attributes. There is a notable difference between CCFL and LED backlight;

- The low price, middle size and carbon fiber case have notably high utilities. On the other hand, most 
Step 0: Input module

Input data: STATUTIK $\mathrm{I}_{\mathrm{I}}$ PRODUTIL

Step 1: Initialization module

Generate an initial population of candidate product line extension schemes.

Store the population in matrix $P O P_{M^{*} N^{*} K}$.

\section{Step 2: Evaluation module}

Fitness evaluation of the initial population. Compute matrices $A D D P U_{\mathrm{M}^{*} \omega^{*} \mathrm{PN}}$,

$S O C_{P I}, T O T A L_{-} S O C_{P}$, as described in above section The last element of the

sorted matrix TOTAL_SOC $C_{P}$ corresponds to the best candidate scheme.

\section{Step 3: Sorting module}

Sorting of the elements of matrix TOTAL_SOC and selection of the best candidate scheme of the initial population.

Step 4: New population generation module

\subsection{Selection}

Choose the (40\%) M best candidate schemes. They choose the elements corresponding to the (40\%) $\mathrm{M}$ last elements of the sorted matrix TOTAL_SOC ${ }_{M}$.

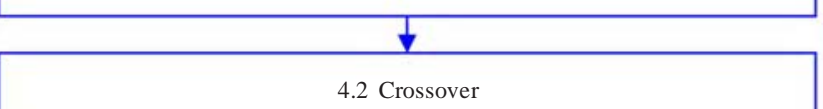

Create randomly (20\%)M pairs of candidate schemes chosen among the ones created in $(40 \%) \mathrm{M}$ best candidate schemes of step 4.1. Perform the uniform crossover operator on these pairs to generate $(40 \%) \mathrm{M}$ new candidate schemes.

\subsection{Mutation}

Pick randomly (20\%)M candidate schemes from the set of the (80\%)M candidate schemes which were created in steps 4.1 and 4.2 . Alter randomly the value at a random string position of each candidate scheme.

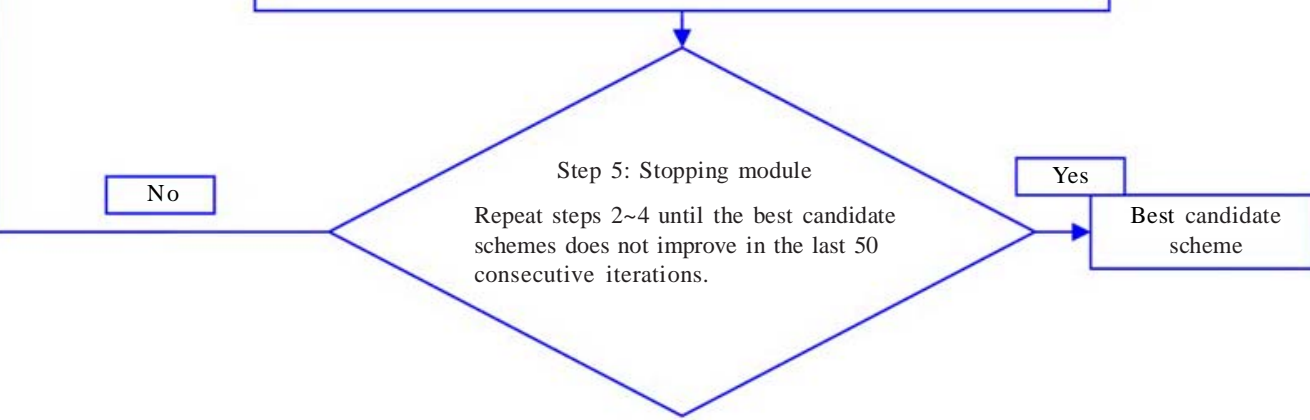

Fig. 3: Solution procedure of proposed GA 
consumers prefer these levels to others in the same attribute. On the contrary, high price, small size and plastic case attributes have notably low utilities.

\section{Results of PLES}

Taking the competition between the existing products of competitors and the existing products of the example company as its basis, this study uses Niche GT that can be commercialized as NB attributes to evaluate how to design a new PLES based on the existing product line in Taiwan. The results of this model were showed in Table 3. These results put particular emphasis on the 14, 17 and 11 inch size. Each product of Table 3 includes some Niche GT and the price is high. The newly added PLES would ideally include one or two products, and the market-expanding capability of each single product is good. Some new technologies showed good market expansion results, such as LED backlights, LP batteries and SSD disks. The new products combined bio-based plastic, whose market expansion capabilities are poor, and this technology does not appear in the following scheme of product line extension. The benefit of bio-based plastic brought by market expansion is marginal compared with the cost of technology adoption.
Table 2: Part-worth utilities of each attribute level

\begin{tabular}{llc}
\hline Attribute & Level & $\begin{array}{c}\text { Part-worth } \\
\text { utilities }\end{array}$ \\
\hline Case & plastic & -0.219 \\
& carbon fiber & 0.255 \\
& metal & 0.093 \\
Batteries & bio-based plastic & -0.130 \\
& lithium ion & 0.043 \\
Backlights & lithium polymer & -0.043 \\
& CCFL & -0.098 \\
Hard disk & LED & 0.098 \\
Size & HDD & -0.034 \\
& SSD & 0.034 \\
& 7 & -0.443 \\
Price & 11 & 0.238 \\
& 14 & 0.173 \\
& 17 & 0.032 \\
& 20000 & 0.803 \\
& 70000 & -0.087 \\
& 120000 & -0.716 \\
\hline
\end{tabular}

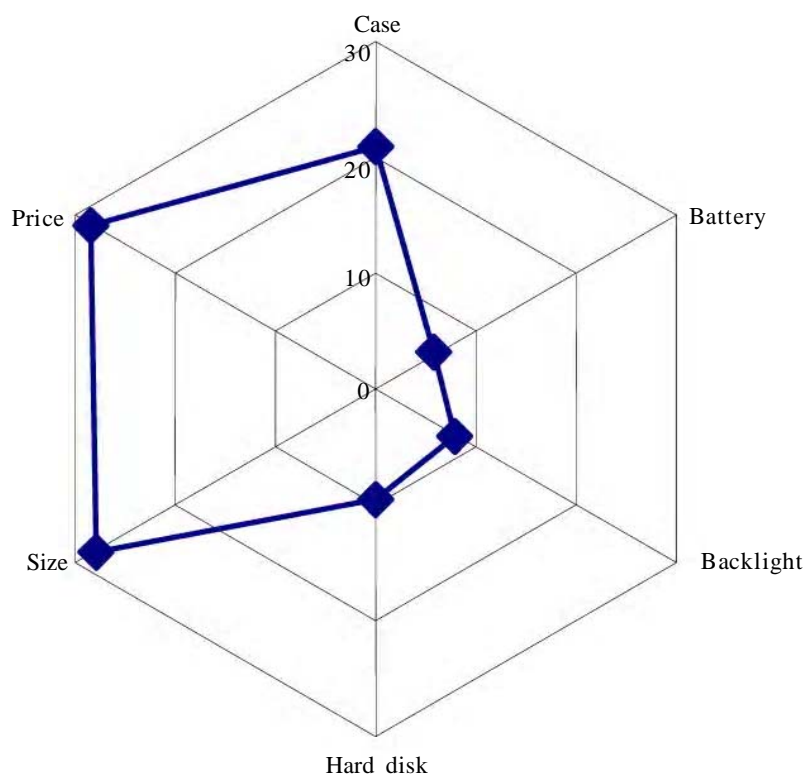

Fig. 4: Importance of each attribute

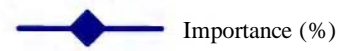


K. H. Lin et al.

Table 3: Increment of market share and profiles of each product in each PLES

\begin{tabular}{|c|c|c|c|c|c|c|c|c|c|}
\hline \multirow[b]{2}{*}{ Scenario } & \multirow{2}{*}{$\begin{array}{l}\text { Index } \\
\text { of } \\
\text { PLES }\end{array}$} & \multicolumn{6}{|c|}{ Attributes and levels } & \multirow{2}{*}{$\begin{array}{c}\text { IMS of } \\
\text { product } \\
(\%)\end{array}$} & \multirow{2}{*}{$\begin{array}{c}\text { IMS of } \\
\text { PLES } \\
(\%)\end{array}$} \\
\hline & & $\begin{array}{l}\text { Size } \\
\text { (inch) }\end{array}$ & Case & Battery & Backlight & $\begin{array}{c}\text { Hard } \\
\text { disk }\end{array}$ & $\begin{array}{c}\text { Price } \\
\text { (NTD) }\end{array}$ & & \\
\hline Extending one product & S1-I & 14 & Metal & LP & LED & HDD & 53000 & 5.7 & 5.7 \\
\hline \multirow[t]{7}{*}{ Extending two products } & \multirow{2}{*}{ S1-II } & 14 & Metal & LP & LED & HDD & 53000 & 5.7 & \multirow{2}{*}{8.6} \\
\hline & & 17 & Metal & LI & LED & SSD & 85000 & 2.9 & \\
\hline & S1-III & 14 & Metal & LP & LED & HDD & 53000 & 5.7 & 8.6 \\
\hline & \multirow{2}{*}{ S1-IV } & 14 & Metal & LP & LED & HDD & 53000 & 5.7 & \multirow{2}{*}{8.6} \\
\hline & & 15 & Metal & LI & CCFL & SSD & 56000 & 2.9 & \\
\hline & \multirow{2}{*}{ S1-V } & 14 & Metal & LP & LED & HDD & 53000 & 5.7 & \multirow{2}{*}{8.6} \\
\hline & & 17 & Metal & LP & CCFL & SSD & 71000 & 2.9 & \\
\hline
\end{tabular}

IMS: increment of market share; PLES: Product line extension scheme; CF: carbon fiber; LP: lithium polymer; LI: lithium ion

\section{CONCLUSION}

- For Niche GT evaluation, this study collects consumer preferences in NB products by CA. In addition, the purchase tendency of individual consumer can be forecasted according to part-worth utilities and establish a PLDM to achieve the purpose of product line extension. The PLDM is to maximize the number of consumers who change the purchase tendency from the competitors' product to the candidate products of the PLSE. The conclusions of this study are as follows:

- For NB companies in Taiwan which are known for the flexibility in product design and manufacturing, proficient utilization of Niche GT in designing PLES can not only expand market but also enhance brand image. NB companies of Taiwan may gain immediate competition advantages if they can promptly reflect consumers' preferences in the design and manufacturing of their products;

- The results of this study include analysis on GT of $\mathrm{NB}$, investigation of consumer preferences and suggestions of PLES; it offers companies both a reference program and a method to appraise product line extension. This study helps NB companies to decide whether or not to adopt Niche GT and how to match with other technologies. This research process can be used on other industries which have Niche GT;

- For products with 14 inch sizes, matching Niche GT such as LED backlights and LP batteries can expand the market. These products can attract the consumers whose original choice is competitors' existing product. This result can help the Example
Company form strategy for Niche GT development sequencing and the matching products;

- For operating practice, the number and the characteristics of participators in survey should be paid more attention and then different segments of market can be considered in this study. Furthermore, the incremental cost evaluation of teach Niche GT will also influence the results of product line design. Company must attach importance to above factors in operating practice.

\section{Nomenclature}

PLES: product line extend scheme

$K: k \in \Omega(=\{1,2, \ldots, K\})$ denotes the set of $K$ attributes $j: j \in \Phi_{k}\left(=\left\{1,2, \ldots, J_{k}\right\}\right)$ denotes the set of $J_{k}$ levels of attribute $k$

$i: \quad i \in \Theta(=\{1,2, \ldots, I\})$ denotes the set of $I$ customers $m: m \in \Psi(=\{1,2, \ldots, M\})$ denotes the set of $M$ items in PLES

$p: p \in \Pi(=\{1,2, \ldots, P\})$ the set of the candidate PLESs STATUK $_{I}$ : a matrix that maintains the part worth utilities of the competitors' existing product which has maximal preference for each customer $i \in \Theta$.

PRODUTIL $_{I}$ : a matrix that maintains the part worth utilities of example company's existing product which has maximal preference for each customer $i \in \Theta$.

$P O P_{I^{*} M^{*} K}$ : a matrix that maintains the population, The elements $P O P_{p m k}$, where $p \in \Pi, m \in \Psi$ and $k \in \Omega$, denote the selected level of each attribute.

$A D D P U_{I^{*} I^{* M}}:$ a matrix that maintains the utilities of the different products of each candidate PLES which are derived by each customer. 
$S O C_{P I}$ : a matrix that maintains customer who originally choose competitors' existing product would change to buy one item of the PLES

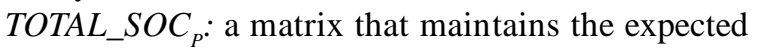
share-of-choices

\section{REFERENCES}

Alexouda, G., (2002). An evolutionary algorithm based method for the product line design using the share of choices criterion. Second Hellenic Conference on Artificial Intelligence, 321-330.

Alexouda, G., (2004). An evolutionary algorithm approach to the share of choices problem in the product line design. Comput. Oper. Res., 31 (13), 2215-2229 (15 pages).

Alexouda, G., (2005). An user-friendly marketing decision support system for the product line design using evolutionary algorithms. Decis. Supp. Sys., 38 (4), 495509 (15 pages).

Alexouda, G.; Paparrizos, K., (1999). A genetic algorithm approach to the buyer's welfare problem of product line design: An comparative computational study. Yugoslav J. Oper. Res., 9 (2), 223-233 (11 pages).

Alexouda, G.; Paparrizos, K., (2001). A genetic algorithm approach to the product line design problem using the seller's return criterion: An extensive comparative computational study. Eur. J. Oper. Res., 134 (1), 165-178 (14 pages).

Balakrishnan, P. V.; Gupta, R.; Jacob, V. (2004). Development of hybrid genetic algorithms for product line designs. IEEE T. Syst. Man Cyb., 34 (1), 468-483 (16 pages).

Balakrishnan, P. V.; Jacob, V. S., (1996). Genetic algorithms for product design. Manage. Sci., 42 (8), 1105-1117 (13 pages).

Bandyopadhyay, G.; Chattopadhyay, S., (2007). Single hidden layer artificial neural network models versus multiple linear regression model in forecasting the time series of total ozone. Int. J. Environ. Sci. Tech., 4 (1), 141-150 (10 pages)

Chen, C. C., (2009). Environmental impact assessment framework by integrating scientific analysis and subjective perception. Int. J. Environ. Sci. Tech., 6 (4), 605-618 (14 pages).

Chien, M. K.; Shih, L. H., (2007). An empirical study of the implementation of green supply chain management practices in the electrical and electronic industry and their relation to organizational performances. Int. J. Environ. Sci. Tech., 4 (3), 383-394 (12 pages).

Dobson, G.; Kalish, S., (1988). Positioning and pricing a product line. Marketing Sci., 7 (2), 107-125 (19 pages).

Green, P. E.; Krieger, A. M., (1985). Models and heuristics for product line selection. Market. Sci., 4 (1) 1-19 (19 pages).

Green, P. E.; Krieger, A. M., (1989). Recent contribution to optimal product positioning and buyer segmentation. Eur. J. Opl. Res., 41 (2), 127-141 (15 pages).

Green, P. E.; Krieger, A. M., (1991). Product Design Strategies for Target Market Positioning. J. Prod. Innovat. Manage., 8 (3), 189-202 (14 pages).
Green, P. E.; Srinivasan, V., (1978). Conjoint analysis in consumer research: Issues and outlook. J. Cons. Res., 5 (2), 103-123 (21 pages).

Gross, R. A.; Kalra, B., (2002). Biodegradable polymers for the environment. Science, 297 (5582), 803-807 (5 pages)

Hsu, C. W.; Hu, A. H., (2008). Green supply chain management in the electronic industry. Int. J. Environ. Sci. Tech., 5 (2), 205-216 (12 pages).

Huang, P. S.; Shih, L. H., (2009). Effective environmental management through environmental knowledge management. Int. J. Environ. Sci. Tech., 6 (1), 35-50 (16 pages).

Kang, Y.; Lee, W.; Suh, D. H.; Changjin, L., (2003). Solid polymer electrolytes based on cross linked-polysiloxaneg-oligo (ethylene oxide): Ionic conductivity and electrochemical properties. J. Power. Sour., 119-121, 448453 (6 pages).

Kohli, R.; Sukumar, R., (1990). Heuristics for product-line design using conjoint analysis. Manage. Sci., 36 (12), 14641477 (14 pages).

Krieger, A. M.; Green, P. E.; Wind, Y. J., (2004). Adventures in conjoint analysis: A practitioner's guide to trade-off modeling and applications. Monograph, University of Pennsylvania.

Li, H.; Azarm, S., (2002). An approach for product line design selection Under Uncertainty and Competition. J. Mech. Design, 124(3), 385-392 (8 pages).

Masuda, Y.; Nakayama, M.; Wakihara, M., (2007). Fabrication of all solid-state lithium polymer secondary batteries using PEG-borate/aluminate ester as plasticizer for polymer electrolyte. Solid. State. Ionics., 178 (1314), 981-986 (6 pages).

Nair, S. K.; Thakur, L. S.; Wen, K. W., (1995). Near optimal solutions for product line design and selection: Beam search heuristics. Manage. Sci., 41 (5), 767-785 (19 pages).

Nnorom I. C.; Osibanjo O., (2009). Heavy metal characterization of waste portable rechargeable batteries used in mobile phones. Int. J. Environ. Sci. Tech., 6 (4), 641-650 (11 pages).

Steiner, W.; Hruschka, H., (2003). Genetic algorithms for product design: How well do they really work? Int. J. Market. Res., 45 (2), 229-240 (12 pages).

Szymanski, D.; Bharadwaj, S.; Varadarajan, R. (1993). An analysis of the market share profitability relationship. J. Market., 29, 1-18 (18 pages).

Tehrani, S. M.; Karbassi, A. R.; Ghoddosi, J.; Monavvari, S. M.; Mirbagheri, S. A., (2009). Prediction of energy consumption and urban air pollution reduction in eshopping adoption. J. Food, Agri. Environ., 7 (3 and 4), 898-903 (7 pages).

Tsai, H. C., (2007). An investigation into EMI-induced noise in nanometer multi-quantum well InGaN LEDs. Opt. Commun., 273 (2), 311-319 (9 pages).

Tuzkaya, G.; Gülsün, B., (2008). Evaluating centralized return centers in a reverse logistics network: An integrated fuzzy multi-criteria decision approach. Int. J. Environ. Sci. Tech., 5 (3), 339-352 (15 pages). 
Wittink, D. R.; Cattin, P., (1989). Commercial use of conjoint analysis: An update. J. Marketing., 53 (3), 91-96 (6 pages).
Zufryden, F. S., (1979). ZIPMAP-A zero-one integer programming model for market segmentation and product positioning. J. Oper. Res. Soc., 30 (1), 63-70 (8 pages).

\section{AUTHOR (S) BIOSKETCHES}

Lin, K. H., Ph.D. Candidate, Department of Resources Engineering, National Cheng Kung University, Tainan, Taiwan. Email: n4892126@ccmail.ncku.edu.tw

Shih, L. H., Ph.D., Full professor, Chairperson, Department of Resources Engineering, National Cheng Kung University, Tainan, Taiwan. Email: Ihshh@mail.ncku.edu.tw

Lee, S. C., Ph.D. Candidate, Department of Resources Engineering, National Cheng Kung University, Tainan, Taiwan. Email: n4895102@mail.ncku.edu.tw

\footnotetext{
How to cite this article: (Harvard style)

Lin, K. H.; Shih, L. H.; Lee, S. C., (2010). Optimization of product line design for environmentally conscious technologies in notebook industry. Int. J. Environ. Sci. Tech., 7 (3), 473-484.
} 